\title{
Behavioural Modelling of Memristive Devices Targeted to Sensor Interfaces
}

\author{
Olufemi A. Olumodeji ${ }^{1,2}$, Massimo Gottardi ${ }^{1}$ \\ ${ }^{1}$ Integrated Radiation and Image Sensors \\ Fondazione Bruno Kessler (FBK) \\ Trento, Italy \\ Olumodeji, gottardi@fbk.eu \\ ${ }^{2}$ Department of Industrial Engineering \\ University of Trento \\ Trento, Italy
}

\begin{abstract}
This paper describes the modelling and application of memristive systems in sensing technology. The proposed architecture is the adoption of memristive devices in place of laser trimming calibration technique to customise a sensor interface. Memristors can also work in combination with analogue and digital electronics to build adaptive systems. Several approaches have been made to overcome some of the disadvantages of laser trimming calibration such as replacing the trimmable resistors with programmable resistor arrays. The ability of the memristor to hold several resistance states can be exploited in an instrumentation amplifier having the memristor as a programmable feedback instead of an array of programmable resistors. A compact behavioural model of the memristor has been developed for the modelling and the simulation of complex circuits for sensor calibration. The model approximates the measurements of the memristor carried out in our labs, and also to simulate memristive logic gates.
\end{abstract}

Keywords - sensor calibration; memristor modelling; memory; Instrumentation amplifier; organic memristor; logic gates; switched memristor;

\section{INTRODUCTION}

For over many years, the resistor, the capacitor and the inductor have been accepted as the only three fundamental passive circuit elements.

In 1971 Dr Leon Chua theorized that mathematically there should be a fourth fundamental circuit element based on the symmetry of the equations that govern passive circuit theory [2]. Dr Chua called this device the memristor (short for memory-resistor), and in 2008 Hewlett Packard Labs published results of the device in physical form [1].

The physical memristor is nanoscale device that has unique properties that can be used to greatly improve existing electronic systems and computing architectures. The memristor can be thought of as a time varying resistor where the

This work was supported by the MaDEleNA project financed by the Provincia Autonoma di Trento, call 'Grandi Progetti 2012'. resistance changes due to the summation of current that has passed through the device [2]. When the current flowing through the device is zero, the summation of current becomes constant, and thus the resistance remains unchanged. This shows that the memristor can be used as a non-volatile memory component. Furthermore, the dynamics of a memristor closely resemble those of a synapse in brain tissue. Just as the values of synaptic weights change with the application of neural spikes, the resistance value of a memristor can be changed with the application of a voltage pulse. This could provide significant advancements in the field of neuromorphic computing as electronic systems using memristors could be fabricated with a device density similar to that of the human brain. Since the memristor's physical discovery, several institutions have published memristor device fabrications using a variety of materials and device structures. Different device structures are still being developed to determine which memristor device would be the best option for commercial use. This is based on many factors such as size, switching speed, power consumption, switching longevity, and CMOS compatibility.

Several device models have also been proposed that can be used to simulate memristor based circuits [7]. The memristor devices, memristor models, and basic circuit designs will serve as the first steps in developing electronic systems and computing architectures based on memristors. A generalised class of devices and systems called memristive devices and systems [8]-[9] was published in 1976 which shows that a large scope of physical systems can be categorised as memristive devices and systems. Since the physical realisation of the memristor, several applications have been proposed for programmable analogue circuits. In this paper we present some application of the memristor in an instrumentation amplifier with programmable gain capability which has the potential of replacing the already existing technology.

This paper is organised in such a way that we introduce the modelling techniques and model fitting with experimental measurements in the next section. Section III introduces the instrumentation amplifier circuit with a programmable feedback memristor. Finally, we conclude in section IV with some discussions. 


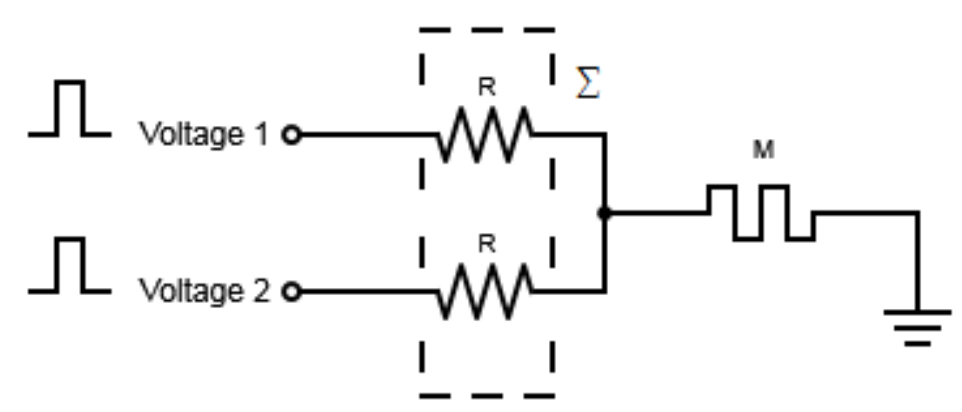

Fig. 1. Schematics of the logic AND gate realisation.
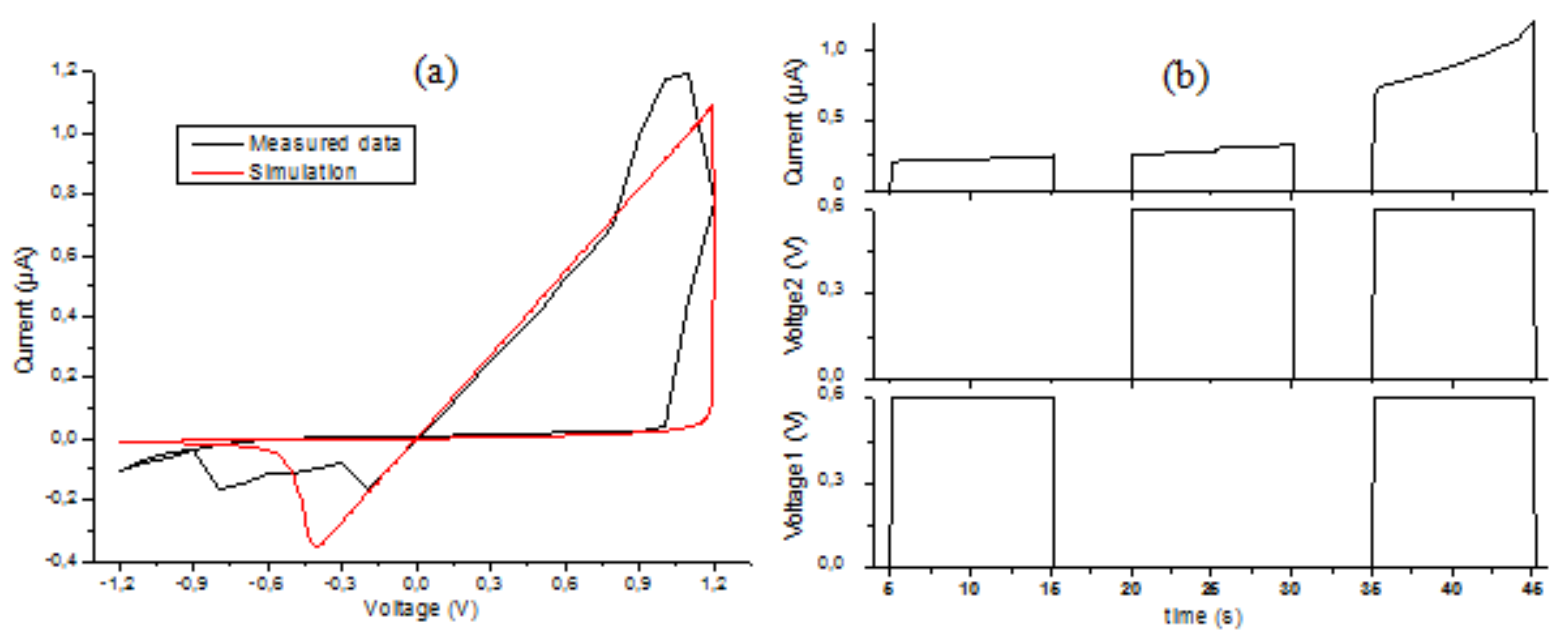

Fig. 2. a, Organic memristor hardware and model fitting with I-V curve. b, Temporal dependence of the output of the MAND function. In (b) the status of the output current for different logic configurations of two inputs. When both inputs are activated, a gradual increase of the output signal can be observed, while the output remains constant when only one input is ON. The value of the output signal depends on the duration of the simultaneously applied input.

\section{MODELLING TECHNIQUES AND MODEL FITTING}

The construction of an accurate and compact model for memristive devices is necessary for the design and modelling of complex adaptive systems. Our goal was to design a behavioural model which approximates the measurements of the memristor carried out in our labs, and also to simulate memristive logic gates. The model has been implemented using Verilog-A, a high-level language that uses modules to describe the structure and behaviour of analogue systems and their components. The advantages of using Verilog-A instead of Spice is that Verilog-A provides a simple, efficient, more concise and clear language for describing analogue behaviour in simulators, simulation run time is much shorter and the implementation in CADENCE [4] which is a standard CAD software for IC design gives the model the advantage of being integrated in a more complex network [9].

Due to the fact that memristive devices share the same common fingerprint as the generalised memristor theorised by Chua in 1971 [1], it is possible to behaviourally model a generalised memristive system (in our case the organic memristor) starting from existing modelling equation of the memristor proposed by HP laboratory. The memristor is modelled as a thin film of two variable resistors in series where the resistance of each is dependent on the doping ratio $w(t)$ [2].

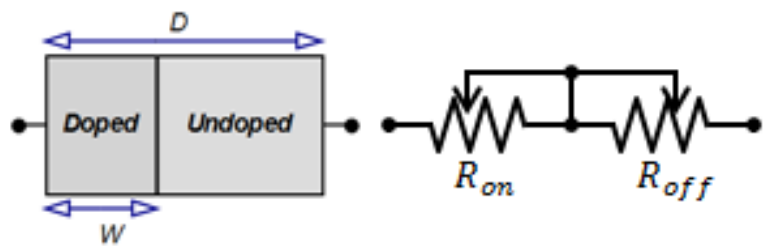

Fig. 3. Memristor device structure and the equivalent circuit model

$$
\begin{gathered}
v(t)=\left(R_{o n} \frac{w(t)}{D}+R_{o f f}\left(1-\frac{w(t)}{D}\right)\right) i(t) \\
\frac{d w(t)}{d t}=\mu_{v} \frac{R_{o n}}{D} i(t)
\end{gathered}
$$

The formula for the doping ration is given in (3).

$$
w(t)=\mu_{v} \frac{R_{o n}}{D} q(t)
$$

For $R_{\text {on }} \ll R_{\text {off }}$, the memristance can be expressed as in (4). 


$$
M(q)=R_{o f f}\left(1-\frac{u_{v} R_{o n}}{D^{2}} q(t)\right)
$$

Even though it has been argued that the pinched hysteresis loop is not a circuit model because both the shape and the area enclosed by the hysteresis lobes change with the input signal, and therefore cannot be used to predict the solution waveforms when the device is embedded as part of an electronic circuit [5]. It is important to note that understanding how the memristor reacts when driven by a voltage can nevertheless help us understand some certain behaviours of the memristor as will be shown from simulation results of a model of the organic memristor fitted from experimental data carried out in our lab, implementing logic with memory.
Fig. 2,a shows the model-to-hardware correlation fit for the organic memristive device, the black line is the measured data while the red line is the simulated data. The simulation result does not accurately fit the experimental result due to certain characteristics of the device which must be considered such as the stability of the device, early ageing of the device. It is also worth noting that device characteristics varies from one device to another and measurements carried out on the same device varies as well. The model, nevertheless, describes the behaviour of the organic memristor.

After fitting the model with the experimental results, we validated it by simulating an AND gate which was recently implemented in [6]. The schematics of the Memorised-AND (MAND) function is shown in Fig. 2,b.

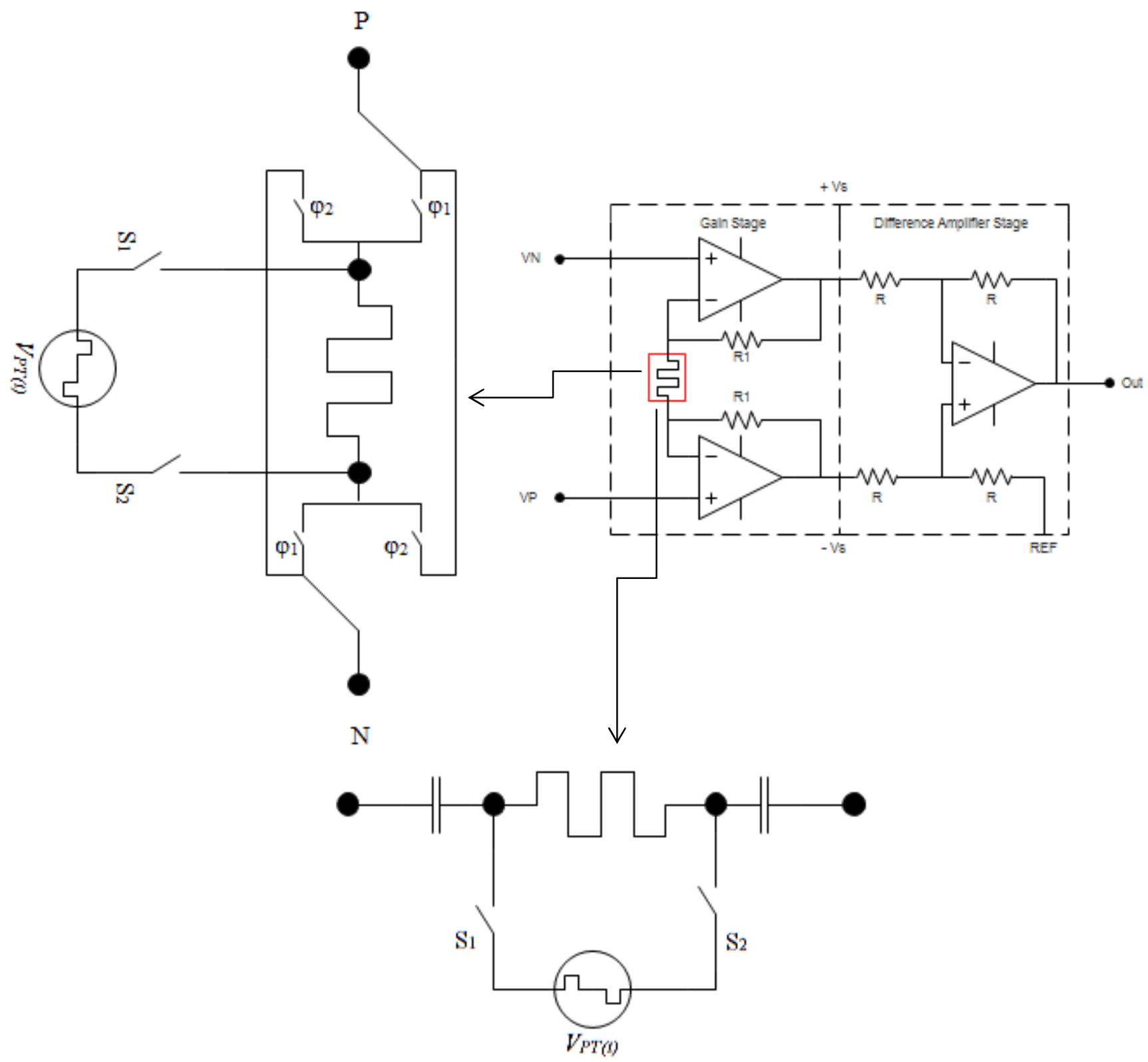

Fig. 4. Schematics of a three operational amplifier configuration of an instrumentation amplifier having the memristor as a programmable feedback. The diagram to the top right is the proposal for DC amplification using chopper technique. The bottom diagram is the already proposed technique for AC amplification in high frequency, coupling the memristor with two capacitors as shown in the schematics. 


\section{INSTRUMENTATION AMPLIFIER APPLICATION IN SENSING TECHNOLOGY}

Basically, sensors produce analogue signal only and almost all sensors require some kind of analogue and signal conditioning or amplification before the signal coming from the sensor can be converted and processed digitally. Instrumentation amplifiers (in-amps) are precision gain blocks that have a differential input and an output that may be differential or single-ended with respect to a reference terminal. These devices amplify the difference between two input signal voltages while rejecting any signals that are common to both inputs.

The instrumentation amplifier is typically a threeoperational amplifier (op-amp) architecture as shown in Fig. 4 [11]. It is consists of a gain stage whose sole purpose is to amplifier the input signal without removing any common mode. The second stage is the difference amplifier stage which removes the common mode without applying any gain.

Given the ability of the memristor to hold several resistance states and the non-volatility of it memory [10], we can take advantage of this in many analogue circuit applications. In our case, we want to be able to vary the gain of the instrumentation amplifier with just a single memristor. Fig. 4 is a schematic of the instrumentation amplifier with gain programmability in both $\mathrm{AC}$ and $\mathrm{DC}$ amplification. Both the $\mathrm{AC}$ and the $\mathrm{DC}$ voltage amplifiers can be implemented without perturbing the system. Even though it can be argued that it is quite easier to implement a good AC voltage amplifier rather than a DC, some applications involving the instrumentation amplifier requires some DC amplification that's why we propose a technique for controlling the flux across the memristor by using the chopping technique in a chopper amplifier [11] as shown in Fig. 4 (top left schematic).

The chopping block in Fig. 4 (top left schematic) is controlled by two non-overlapping phase clock. The parameters for the non-overlapping phases and also of the memristor model has to be chosen in such a way that the net flux across the across in one clock cycle is not enough to change the state of the memristor.

In order to change the gain of the instrumentation amplifier, the memristor can be pulsed from an external generator as described in [12].

\section{CONCLUSION}

A model of the memristive device has been developed with model to hardware fit correlation and tested to be the basic building block to carry out the design phase of mixed analogue/digital electronics based on the memristive properties. The proposed model is based on Verilog-A which is a behavioural language covering analogue design.

The unique behaviour of the memristor and its resistance programmability has also been presented with an application in the instrumentation amplifier targeted for the sensing technology. The instrumentation amplifier is based on two different memristor circuits (one for $\mathrm{AC}$ amplification and the other for DC amplification). In our future work we're going to emphasise more on the switched memristor circuit for DC amplification.

\section{REFERENCES}

[1] Strukov, D. B., Snider, G. S., Stewart, D. R., \& Williams, R. S. (2008). The missing memristor found. nature, 453(7191), 80-83.

[2] Chua, Leon O. "Memristor-the missing circuit element." Circuit Theory, IEEE Transactions on 18.5 (1971): 507-519.

[3] Chua, Leon O. "The fourth element." Proceedings of the IEEE 100.6 (2012): 1920-1927.

[4] Cadence Verilog-A language reference Manual. Version 5.0 Open Verilog International, 2002

[5] Chua, Leon. "Memristors: Past, Present and Future." (2012).

[6] G Baldi, S Battistoni, G Attolini, M Bosi, C Collini, S Iannotta, L Lorenzelli, R Mosca, J S Ponraj, R Verucchi and V Erokhin "Logic with memory: and gates made of organic and inorganic memristive devices." Semicond. Sci. Technol. 29 (2014)

[7] Biolek, Zdeněk, Dalibor Biolek, and Viera Biolkova. "SPICE model of memristor with nonlinear dopant drift." Radioengineering 18.2 (2009): 210-214.

[8] Chua, Leon O., and Sung Mo Kang. "Memristive devices and systems."Proceedings of the IEEE 64.2 (1976): 209-223.

[9] Ventra, M. D. "Memristive Systems: From Spintronics to Amoeba's Learning."Memristor and Memristive Systems Symposium. 2008.

[10] J. J. Yang, M. D. Pickett, X. Li, D. A.Ohlberg, D. R. Stewart, and R. S. Williams, "Memristiveswitching mechanismfor metal/oxide /metal nanodevices," Nature Nanotechnology 3,pp.429-433,2008.

[11] Kolacinski, C., and Dariusz Obrebski. "Design of CMOS analog integrated readout circuit for NMOS THz detectors." Mixed Design of Integrated Circuits and Systems (MIXDES), 2013 Proceedings of the 20th International Conference. IEEE, 2013.

[12] Shin, Sangho, Kyungmin Kim, and S. Kang. "Memristor-based fine resolution programmable resistance and its applications." In Communications, Circuits and Systems, 2009. ICCCAS 2009. International Conference on, pp. 948-951. IEEE, 2009. 